\title{
Rapid Treatment of Leukostasis in Leukemic Mantle Cell Lymphoma using Therapeutic Leukapheresis: A Case Report
}

\author{
Xuan Duc Nguyen ${ }^{1, \star}$, Paul La Rosée ${ }^{2}$, Thomas Nebe $^{3}$, Harald Klüter ${ }^{1}$, and \\ Dieter Buchheidt ${ }^{2}$ \\ ${ }^{1}$ Institute of Transfusion Medicine and Immunology, Medical Faculty Mannheim, \\ University of Heidelberg, Red-Cross Blood Service of Baden-Württemberg - \\ Hessen, Germany; ${ }^{2}$ III. Medical Clinic, Medical Faculty Mannheim, University of \\ Heidelberg, Germany; ${ }^{3}$ Institute for Clinical Chemistry, Medical Faculty Mannheim, \\ University of Heidelberg, Germany \\ E-mail: xuan-duc.nguyen@medma.uni-heidelberg.de
}

Received June 15, 2011; Revised July 27, 2011; Accepted July 27, 2011; Published August 16, 2011

We describe a case of severe leukocytosis caused by leukemic mantle cell lymphoma (MCL), complicated by leukostasis with myocardial infarction in which leukapheresis was used in the initial management. A 73-year-old male presented to the emergency department because of fatigue and thoracic pain. Blood count revealed $630 \times 10^{9} / \mathrm{L} \mathrm{WBC}$ (white blood cells). The electrocardiogram showed ST-elevation with an increase of troponin and creatinine kinase. The diagnosis was ST-elevation myocardial infarction (STEMI) induced and complicated by leukostasis. Immunophenotyping, morphology, cytogenetic and fluorescence-in-situ-hybridization analysis revealed the diagnosis of a blastoid variant of MCL. To remove leukocytes rapidly, leukapheresis was performed in the intensive care unit. Based on the differential blood count with $95 \%$ blasts, which were assigned to the lymphocyte population by the automatic hematology analyzer, leukapheresis procedures were then performed with the mononuclear cell standard program on the Spectra cell separator. The patient was treated with daily leukapheresis for 3 days. The WBC count decreased to $174 \times 10^{9} / \mathrm{L}$ after the third leukapheresis, with a $72 \%$ reduction. After the second apheresis, treatment with vincristine, cyclophosphamide, and prednisolone was started. The patient fully recovered in the further course of the treatment. To the best of our knowledge, this is the first report on blastoid MCL with leukostasis associated with a STEMI that was successfully treated by leukapheresis. Effective harvest of circulating lymphoma cells by leukapheresis requires adaptation of instrument settings based on the results of the differential blood count prior to apheresis.

KEYWORDS: mantle cell lymphoma, hyperleukocytosis, leukostasis, therapeutic leukapheresis 


\section{INTRODUCTION}

Rapid reduction in the number of circulating blast cells is essential when treating patients with acute hyperleukocytosis (white blood cell [WBC] counts $>100,000 / \mathrm{mm}^{3}$ ). Many early complications and death can be directly attributed to hyperleukocytosis and its resultant microcirculatory dysfunction, a phenomenon referred to as leukostasis[1,2,3]. Leukapheresis has been widely used in the treatment of acute hyperleukocytosis because of its immediate cytoreductive effect[3,4,5,6]. However, patients with lymphoid malignancies rarely develop leukostasis, but may undergo cytoreduction with leukapheresis as prophylaxis for tumor lysis[6,7]. Here, we report a case of severe leukocytosis caused by a mantle cell lymphoma (MCL), a blastoid variant, complicated by leukostasis in which leukapheresis was used in the initial management. A blastoid variant of MLC is a morphologic variant characterized by large, noncleaved cells with fine chromatin and inconspicuous nucleoli. Reports of MCL with blastoid variant, defined as seen in the current case, are rare[8,9,10,11]. Until now, only one prior report described a blastoid MCL with a leukocyte count of $405 \times 10^{9} / \mathrm{L}[10]$. To the best of our knowledge, our study represents the first reported case of a leukemic MCL, blastoid variant, with a hyperleukocytosis of $630 \times$ $10^{9} / \mathrm{L}$, complicated with leukostasis causing a myocardial infarction (MI). Leukapheresis was successfully performed for a rapid reduction of circulating lymphoma cells, resulting in an improvement of the patient's condition.

\section{CASE REPORT AND RESULTS}

A 73-year-old male presented to the emergency department because of fatigue and chest pain. He had no fever, chills, or night sweats. He had a history of peripheral artery occlusive disease and coronary heart disease, with coronary angioplasty and stent implantation in 1998. On physical examination, he appeared fatigued and had a mild dyspnea. The blood pressure, pulse, and oxygen saturation were at 160/85 mm, 90 beats per minute, and 95\%, respectively. His lungs were clear to auscultation. He had no splenomegaly, adenopathy, hepatomegaly, or skin lesions. Differential blood count and morphology revealed an extreme leukocytosis of $600 \times 10^{9} / \mathrm{L} \mathrm{WBC}$, with $95 \%$ lymphoma cells assigned as mononuclear cells (MNC)(Fig. 1). Hematocrit (Hct) and platelet count were at $27 \%$ and $108 \times 10^{9} / \mathrm{L}$, respectively. Laboratory investigations revealed elevated values of $\gamma$ - GT, ASAT, AP, LDH, uric acid, CRP, and fibrinogen with $93 \mathrm{U} / \mathrm{L}$ (0-55), $96 \mathrm{U} / \mathrm{L}$ (0-37), $341 \mathrm{U} / \mathrm{L}$ (38-126), $795 \mathrm{U} / \mathrm{L}$ (0-248), $10.4 \mathrm{mg} / \mathrm{dL}$ (2.5-5.7), $92 \mathrm{mg} / \mathrm{L}(0-5)$, and $6.01 \mathrm{~g} / \mathrm{L}$ (1.5-4.5), respectively. Measurements of the electrolytes, coagulation indices (PTT, INR), creatinine, cardiac enzymes CPK, and troponin-I were normal. The patient was admitted to the intensive care unit for further treatment. Based on the electrocardiogram and the cardiac enzymes at the time of admission to the intensive care unit, MI could be excluded. To remove leukocytes rapidly, therapeutic leukapheresis was immediately induced after the admission of the patient to the intensive care unit. In order to choose the appropriate collection program on the Spectra cell separator for the effective harvest of circulating lymphoma cells, a differential blood count was performed before apheresis using an automatic hematology analyzer and revealed a further increase of WBC with $630 \times 109 / \mathrm{L}$ differentiated in neutrophils $1 \%$, lymphocytes $88 \%$, monocytes $8 \%$, and eosinophils $3 \%$. Due to the fact that lymphoma cells were assigned to the lymphocyte and monocyte population by the hematology analyzer, leukapheresis procedures were then performed with the MNC standard program on the cell separator. The total volume of leukocyte-rich plasma removed ranged from 513-600 $\mathrm{mL}$ per treatment. Each apheresis required $2-3 \mathrm{~h}$ to complete. After the first leukapheresis, the WBC count decreased to $409 \times 10^{9} / \mathrm{L}$. Prior to the second apheresis, the WBC count increased to $541 \times 10^{9} / \mathrm{L}$. At that time, the patient reported dyspnea. The electrocardiogram showed a ST-elevation in the ECG channels V1-V4 and a negative T wave in channels I, II, and aVF. The values of troponin-I and CPK increased to $0.8 \mu \mathrm{g} / \mathrm{L}(0-0.5)$ and $207 \mathrm{U} / \mathrm{L}(0-145)$, respectively. Thus, the diagnosis was ST-elevation myocardial infarction (STEMI) induced and complicated by leukostasis. Because of the reduced physical status of the 


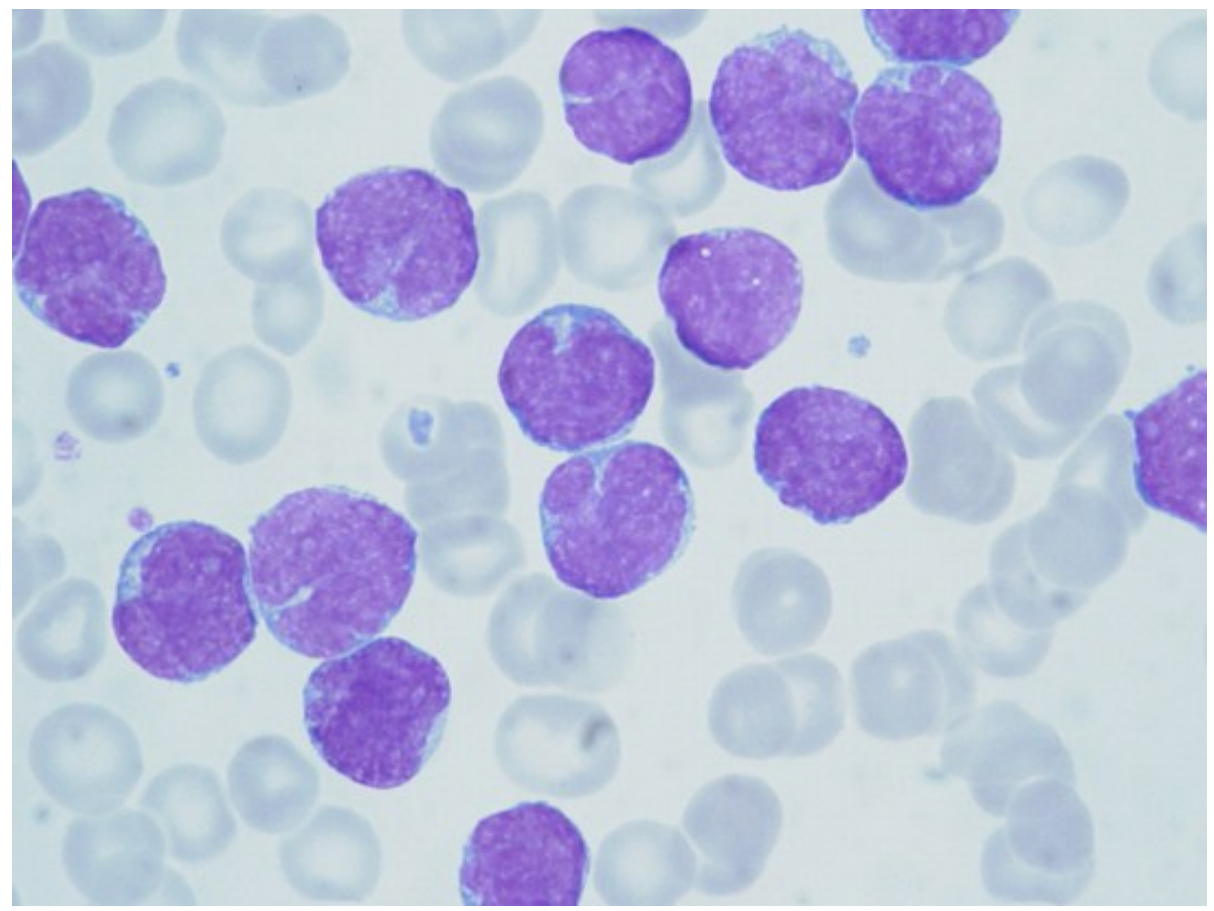

FIGURE 1. Peripheral blood smear. A population of mantle cells with cleaved nucleus and paucity of cytoplasma (May-Grünwald-Giemsa staining, original magnification $\times 1160$ ). The majority of cells in the figure appear cleaved, showing an unusual variant of MCL.

patient, an angioplasty could not be performed. Due to the STEMI, the following apheresis procedures were performed without transfusion of platelets (PLT), while the patient's PLT decreased to $60 \times 10^{9} / \mathrm{L}$ and $35 \times 10^{9} / \mathrm{L}$ prior to the second and third apheresis, respectively (Fig. 2). The patient was treated with daily leukapheresis for 3 days. The WBC count decreased to $174 \times 10^{9} / \mathrm{L}$ after the third leukapheresis, with an elimination rate of $43 \%$ reduction (35-49\%) in WBC count per leukapheresis (Fig. 2). The patient showed a relief of symptoms after the second apheresis, with a decrease of WBC count from $541 \times 10^{9} / \mathrm{L}$ to $305 \times 10^{9} / \mathrm{L}$. The CPK and troponin-I went back to $143 \mathrm{U} / \mathrm{L}(0-145)$ and $0.68 \mu \mathrm{g} / \mathrm{L}(0-0.5)$, respectively. After 1 week, troponin-I and ECG had fully recovered.

According to the immunophenotyping by flow cytometry (3-color machine, Becton Dickinson, Heidelberg, Germany), the lymphoma cells were positive for CD5, CD19, CD20, CD22, CD24, CD25, CD38, CD79, FMC7, and HLA-DR. The cells were monoclonal for lambda with a strong expression of the light chain. The investigation on CD1, CD2, CD3, CD4, CD8, CD10, CD11c, CD13, CD14, CD15, CD16, CD23, CD34, CD56, CD61, CD65, CD103, CD117, CD235, myeloperoxidase, and TdT showed a negative result. Fluorescence-in-situ-hybridization (FISH) was performed using the IGH-CCND1 (t11;14) probe (ABBOTT).In 93 of 100 analyzed peripheral blood interphase cells, the typical colocalization signal for the IGH-CCND1 rearrangement confirming translocation $(11 ; 14)$ was detected.

All the results were consistent with MCL, blastoid variant. Treatment with vincristine (1 mg once), cyclophosphamide (350 mg/die for 5 days), and prednisolone $(100 \mathrm{mg} / \mathrm{die})$ was started after the second leukapheresis. The WBC count dropped to $11 \times 10^{9} / \mathrm{L}$ after 2 weeks. The patient recovered fully and induction chemotherapy was scheduled in the outpatient clinic. Unfortunately, the patient declined further treatments and missed follow-up appointments. 


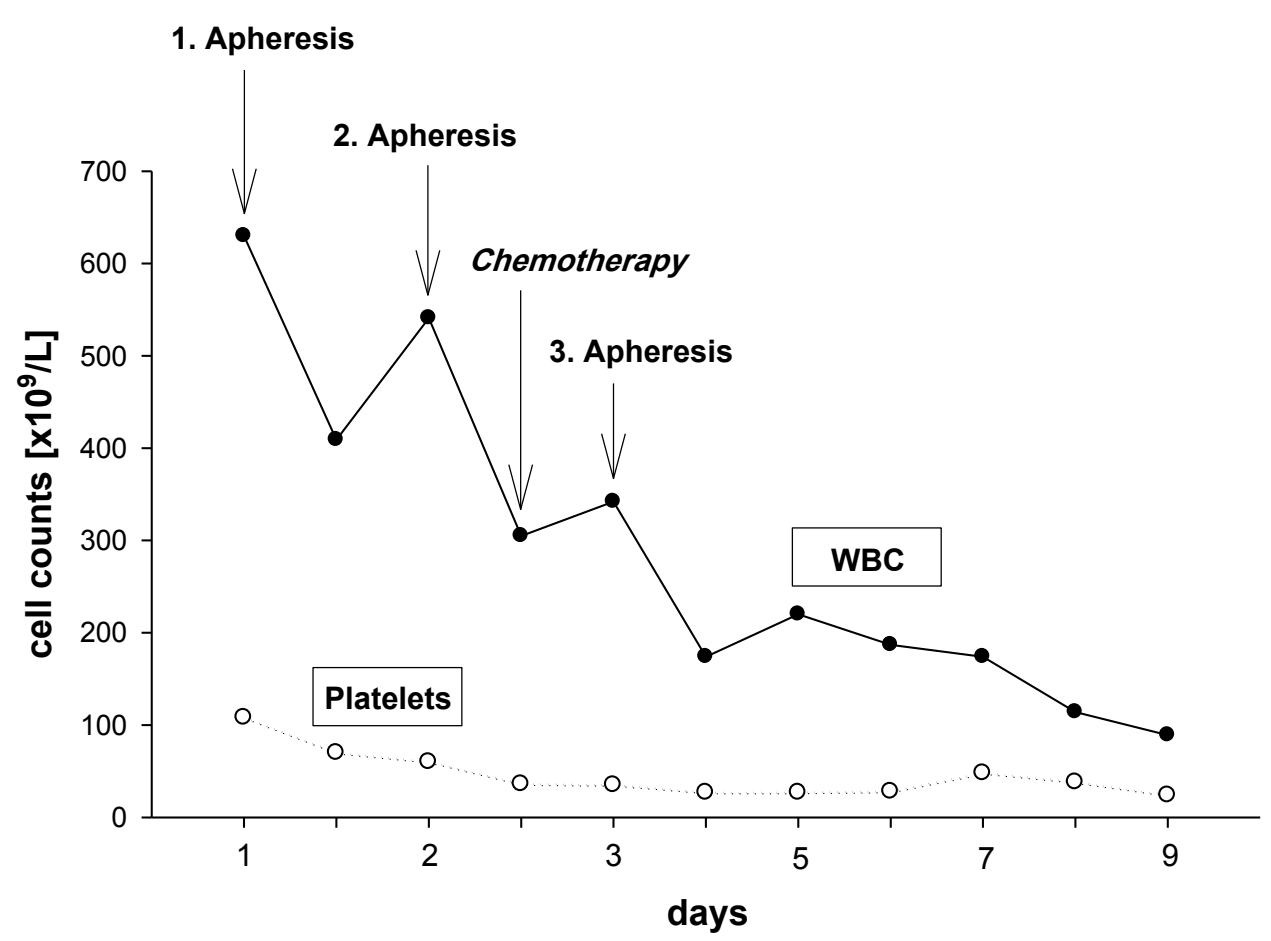

FIGURE 2. WBC count and PLT over time. The patient underwent three sessions of leukapheresis. Chemotherapy with vincristine, cyclophosphamide, and prednisolone was started after the second apheresis.

\section{MATERIALS AND METHODS}

All leukapheresis procedures were performed with the Spectra cell separator (Cobe BCT, Lakewood, CO, USA) by using the V5.1 software and the MNC standard program. The cell separator was set in accordance with the manufacturer's recommendation. Peripheral venous access was performed with cubital venipuncture. ACD-A was used as anticoagulant with an ACD-A blood ratio of 1:15. Wholeblood inlet flow rates of $50 \mathrm{~mL} / \mathrm{min}$ were adjusted by the system according to size of the patient (sex, height, and weight) to reduce the risk of citrate reactions. No adverse reactions during the collections were observed. The collection flow rate was $6 \mathrm{~mL} / \mathrm{min}$, and the plasma pump rate was visually adapted to target a product's Hct of around $2 \%$ by using the color index provided with this apheresis device. No sedimenting agents were added to the blood.

\section{DISCUSSION}

Leukemic MLC shows a very aggressive clinical course and is associated with poor prognosis; survival ranges between 1.5 and 37 months[8,10]. Particularly, blastoid MCL subtypes were characterized by distinctly elevated mitotic counts and proliferation indices[12]. Hyperleukocytosis may result in leukostasis syndrome characterized by impaired flow and accumulation of leukemic cells in the microvasculature. Leukostasis can affect any organ system. Symptoms usually arise from involvement of the pulmonary and cerebral microvasculature, and most early deaths are due to respiratory failure and intracranial hemorrhage[13,14]. Leukapheresis has been used for rapid treatment of acute hyperleukocytosis. Although there are no widely accepted protocols for when to start leukapheresis, it is usually initiated in any patients with AML with a blast count $>100,000 / \mathrm{mm}^{3}$ or in the presence of 
symptoms of leukostasis, irrespective of the blast count[4,14]. However, leukapheresis is rarely performed in ALL or leukemic transformation of lymphomas unless symptoms of leukostasis or blast counts $>300,000 / \mathrm{mm}^{3}$ are seen[14].

Here, we report a case of MCL, blastoid variant, with an extreme leukocytosis complicated with a leukostasis associated with STEMI. The diagnosis was confirmed by morphology, immunophenotyping, cytogenetic and FISH analysis. Only a few cases of AML coincident with acute MI were reported[15,16,17,18] and only one of these reports described cardiac manifestations of leukostasis[15]. Until chemotherapy effectively reduces circulating lymphoma cells, high-volume leukapheresis can be temporarily performed to manage peripheral leukocytosis. Daily treatments may be necessary until the production of WBC can be controlled. Porcu et al. identified older age, respiratory or neurological symptoms, coagulopathy, and renal failure as risk factors for early death in patients with hyperleukocytosis in acute leukemia. This subgroup of patients benefits from prompt and significant leukoreductions[4]. Additionally, the physical removal of a large number of leukemic cells and the ability to administer fresh plasma and electrolytes during a single procedure may significantly reduce the risk of hemorrhage and tumor lysis syndrome[19].

To the best of our knowledge, this is the first report on blastoid MCL with leukostasis associated with a STEMI that was successfully treated by leukapheresis. In order to improve the WBC harvest, we routinely perform a differential blood count before apheresis. The MNC or PMN (polymorphonuclear) standard program was accordingly chosen for the leukapheresis procedure on the cell separator. Due to assignment of the circulating lymphoma cells to the lymphocyte population (mononuclear cell population) by the automatic hematology analyzer, the MNC standard program was selected. Therefore, an erythrocyte sedimentation agent such as hydroxyethyl starch for returning red cells for a better elimination of polymorph cells like granulocytes was not necessary. With the past history of coronary heart disease, the patient was at high risk for MI during leukostasis. A STEMI developed 1 day after admission to the intensive care unit. After the second apheresis with a decrease of the WBC count, the patient showed a complete relief of symptoms correlating with a decrease of CPK and troponin-I. Despite the thrombocytopenia in the patient and the fact of PLT loss due to apheresis[20], transfusion of PLT was not applied, in order to avoid a possible enhancement of clotting that may have aggravated the STEMI. The coagulation indices were normal at the time of apheresis. Nonetheless, peripheral blood PLT levels above $30 \times 10^{9} / \mathrm{L}$ before collection are sufficient for a safe leukapheresis[21]. The reported case demonstrates effective removal of circulating lymphoma cells through leukapheresis, with a $72 \%$ reduction in WBC count. Due to cytoreduction by chemotherapy, three leukapheresis sessions were sufficient.

In conclusion, leukapheresis is an effective and rapid treatment of leukocytosis complicated with leukostasis. It is possible that progression of the leukostasis-associated STEMI was interfered with through continued leukapheresis. Effective harvest of circulating lymphoma cells by leukapheresis requires adaptation of instrument settings based on the results of the differential blood count prior to apheresis.

\section{REFERENCES}

1. van Buchem, M.A., te Velde, J., Willemze, R., and Spaander, P.J. (1988) A underestimated cause of death in leukaemia. Blut 56, 39-44.

2. McKee, L.C., Jr. and Collins, R.D. (1974) Intravascular leukocyte thrombi and aggregates as a cause of morbidity and mortality in leukemia. Medicine 53, 463-478.

3. McCarthy, L.J., Danielson, C.F., and Rothenberg, S.S. (1997) Indications for emergency apheresis procedures. Crit. Rev. Clin. Lab. Sci. 34, 573-610.

4. Porcu, P., Danielson, C.F., Orazi, A., Heerema, N.A., Gabig, T.G., and McCarthy, L.J. (1997) Therapeutic leukapheresis in hyperleucocytic leukaemias: lack of correlation between degree of cytoreduction and early mortality rate. Br. J. Haematol. 98, 433-436.

5. Shafique, S., Bona, R., and Kaplan, A.A. (2007) A case report of therapeutic leukapheresis in an adult with chronic myelogenous leukemia presenting with hyperleukocytosis and leukostasis. Ther. Apher. Dial. 11, 146-149. 
6. Smith, M.D., Singleton, T.P., Balaraman, S., et al. (2004) Case report: mantle cell lymphoma, prolymphocytoid variant, with leukostasis syndrome. Mod. Pathol. 17, 879-883.

7. Blum, W. and Porcu, P. (2007) Therapeutic apheresis in hyperleukocytosis and hyperviscosity syndrome. Semin. Thromb. Hemost. 33, 350-354.

8. Wong, K.F., Chan, J.K., So, J.C., and Yu, P.H. (1999) Mantle cell lymphoma in leukemic phase: characterization of its broad cytologic spectrum with emphasis on the importance of distinction from chronic lymphoproliferative disorders. Cancer 86, 850-857.

9. Singelton, T.P., Anderson, M.M., Ross, C.W., and Schnitzer, B. (1999) Leukemic phase of mantle cell lymphoma, blastoid variant. Am. J. Clin. Pathol. 111, 495-500.

10. Schlette, E., Lai, R., Onciu, M., Doherty, D., Bueso-Ramos, C., and Medeiros, L.J. (2001) Leukemic mantle cell lymphoma: clinical and pathologic spectrum of twenty-three cases. Mod. Pathol. 14, 1133-1140.

11. Smith, M.D., Singleton, T.P., Balaraman, S., Jaiyesimi, I., O’Malley, B., Al-Saadi, A., and Mattson, J.C. (2004) Case report: mantle cell lymphoma, prolymphocytoid variant, with leukostasis syndrome. Mod. Pathol. 17, 879-883.

12. Ott, G., Kalla, J., Ott, M.M., Schryen, B., Katzenberger, T., Müller, J.G., and Müller-Hermelink, H.K. (1997) Blastoid variants of mantle cell lymphoma: frequent bcl-1 rearrangements at the major translocation cluster region and tetraploid chromosome clones. Blood 89, 1421-1429.

13. Pineda, A.A. and Vamakas, E.C. (1997) Applications of therapeutic apheresis in patients with malignant disease. Oncologist 2, 94-103.

14. Porcu, P., Farag, S., Marcucci, G., Cataland, S.R., Kennedy, M.S., and Bissell, M. (2002) Leukocytoreduction for acute leukemia. Ther. Apher. 6, 15-23.

15. Cohen, Y., Amir, G., Da'as, N., Gillis, S., Rund, D., and Polliack, A. (2002) Acute MI as the presenting symptom of acute myeloblastic leukemia with extreme hyperleukocytosis. Am. J. Hematol. 71, 47-49.

16. Candelpergher, G., Suzzi, G.L., Visona, A., and Buchberger, R. (1980) Acute myocardial infarct as the first manifestation of acute myeloid leukemia. Description of an anatomo-clinical case. G. Ital. Cardiol. 10, $1403-1407$.

17. Lisker, S.A., Finkelstein, D., Brody, J.I., and Beizer, L.H. (1967) MI in acute leukemia. Report of a case in a young man. Arch. Intern. Med. 119, 532-535.

18. Solomons, H.D., Stanley, A., King, P.C., Pienaar, N., and Atkinson, P.M. (1986) Acute promyelocytic leukemia associated with acute MI. A case report. S. Afr. Med. J. 70, 117-118.

19. Maurer, H.S., Steinherz, P.G., Gaynon, P.S., Finklestein, J.Z., Sather, H.N., Reaman, G.H., Bleyer, W.A., and Hammond, G.D. (1988) The effect of the initial management of hyperleukocytosis on early complications and outcome of children with acute lymphoblastic leukemia. J. Clin. Oncol. 6, 1425-1432.

20. Nguyen, X.D., Eichler, H., Sucker, A., Hoffmann, U., Schadendorf, D., and Klüter, H. (2002) Collection of autologous monocytes for dendritic cell vaccination therapy in metastatic melanoma patients. Transfusion 42, 428432.

21. Schlenke, P., Frohn, C., Müller-Steinhardt, M., Kirchner, H., and Klüter, H. (2000) Clinically relevant hypokalaemia, hypocalcaemia, and loss of hemoglobin and platelets during stem cell apheresis. J. Clin. Apher. 15, 230-235.

\section{This article should be cited as follows:}

Nguyen, X.D., La Rosée, P., Nebe, T., Buchheidt, D., and Klüter, H. (2011) Rapid treatment of leukostasis in leukemic mantle cell lymphoma using therapeutic leukapheresis: a case report. TheScientificWorldJOURNAL 11, 1554-1559. DOI 10.1100/tsw.2011.142. 


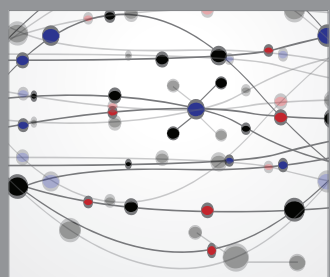

The Scientific World Journal
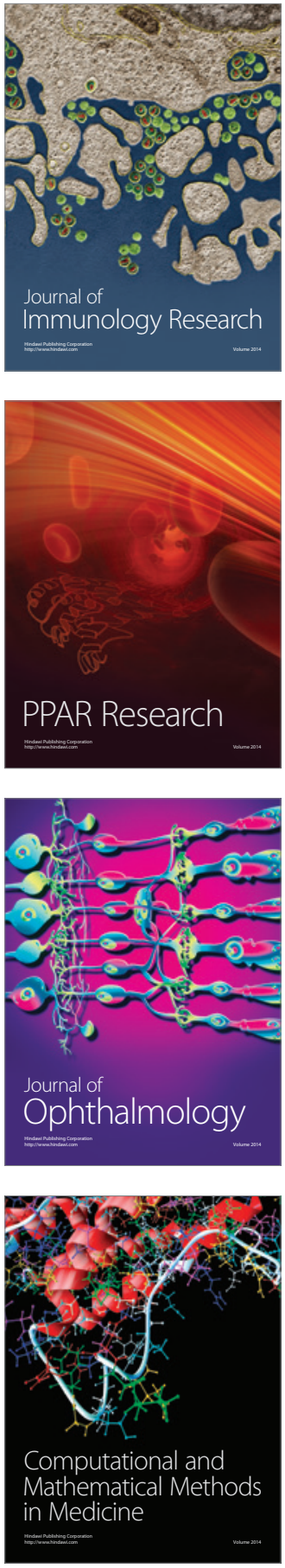

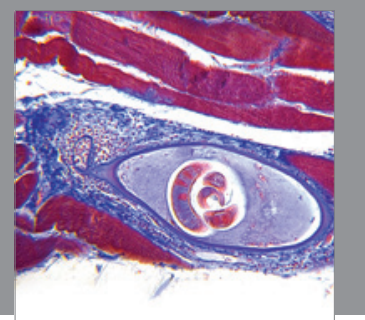

Gastroenterology

Research and Practice
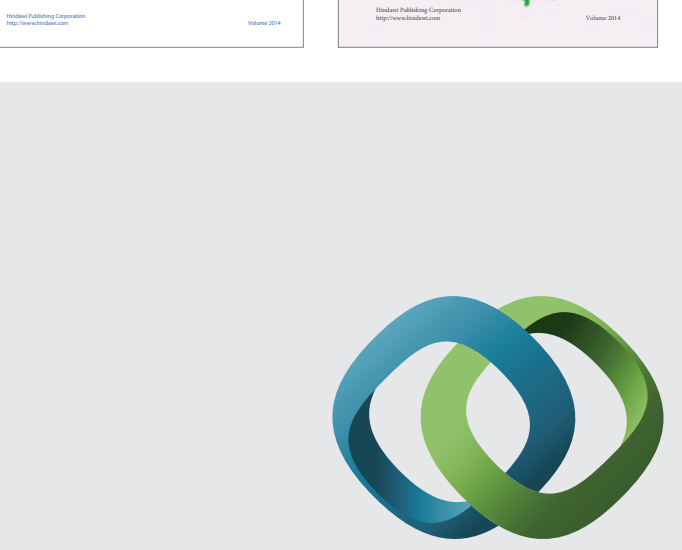

\section{Hindawi}

Submit your manuscripts at

http://www.hindawi.com
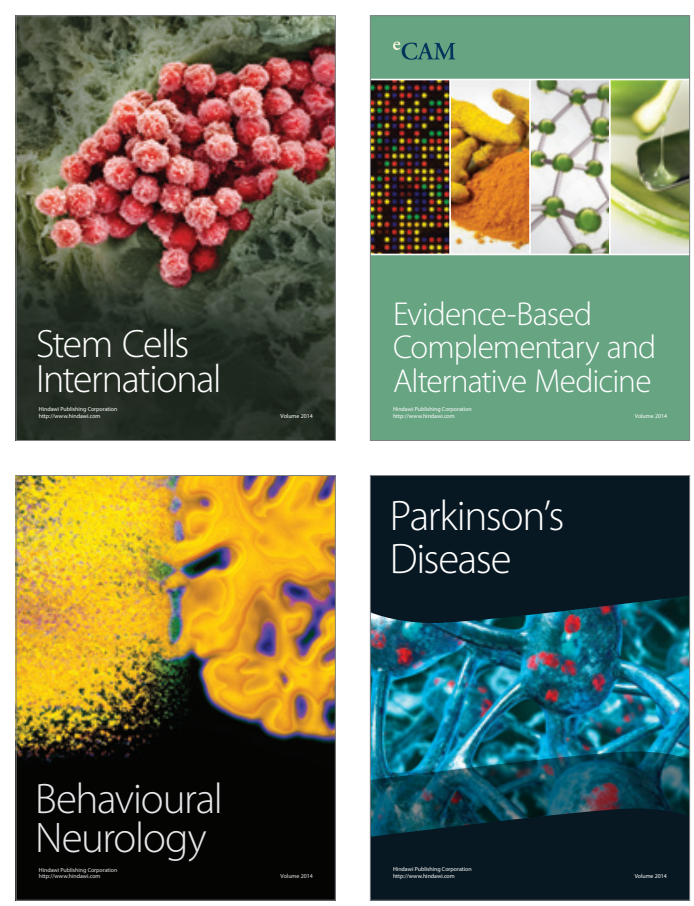

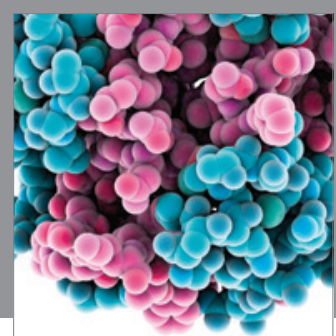

Journal of
Diabetes Research

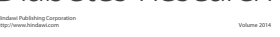

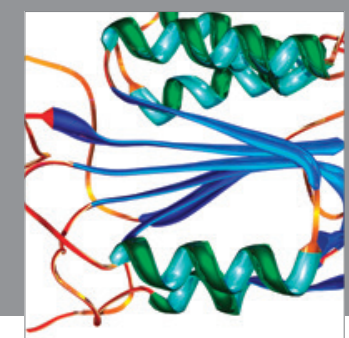

Disease Markers
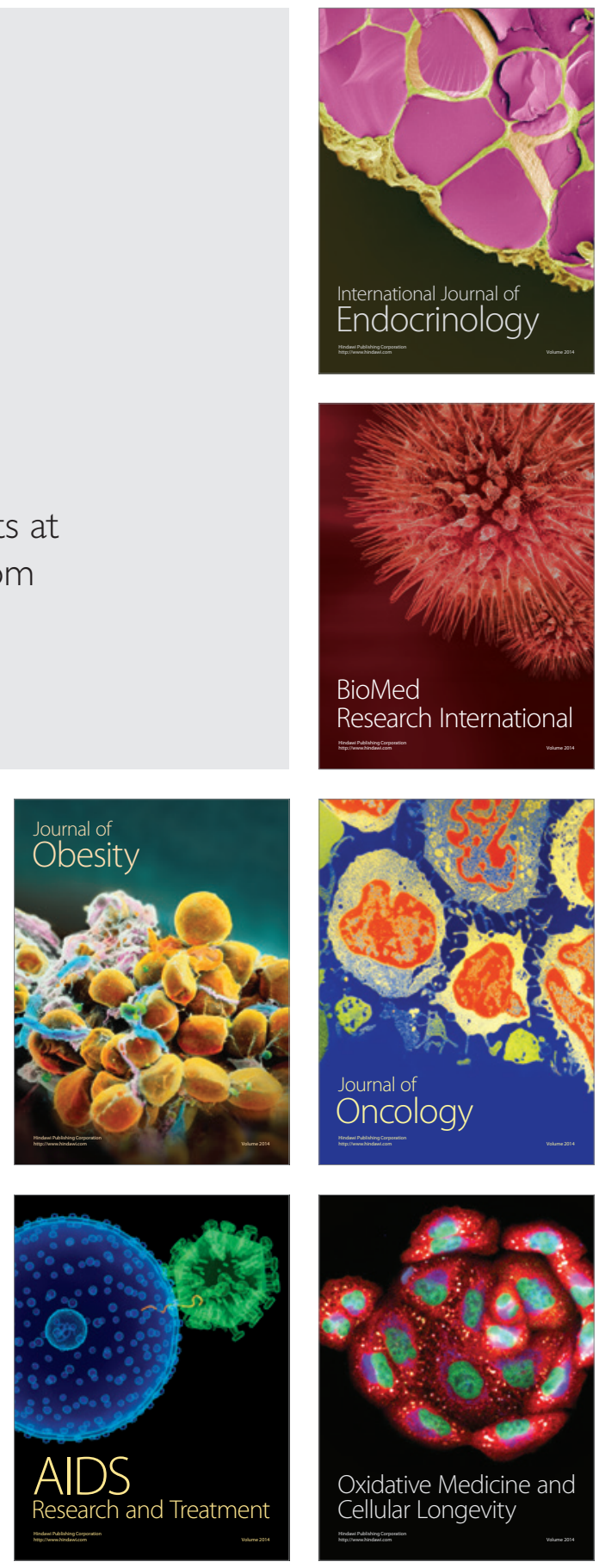